\title{
ASCA SIS X-ray Observations of the Wind Blown Bubble NGC 6888
}

\author{
Matthias Wrigge ${ }^{1}$, You-Hua $\mathrm{Chu}^{2}$, Eugene A. Magnier ${ }^{3}$, and Yuichi Kamata ${ }^{4}$ \\ 1 Hamburger Sternwarte, Gojenbergsweg 112, D-21029 Hamburg, Germany \\ 2 Astronomy Dept., Univ. of Illinois, 1002 W. Green St., Urbana, IL 61801, USA \\ 3 Astronomy Dept., Univ. of Washington, FM-20, Seattle, WA 98195, USA \\ 4 Nagoya University, Furo-cho, Chikusa-ku, Nagoya 464-01 Japan
}

\begin{abstract}
We present ASCA SIS observations of the wind-blown bubble NGC 6888. Because the ASCA SIS is sensitive to higher energy photons and has a higher spectral resolution compared to the ROSAT PSPC, we are able to detect a $T \approx$ $8 \times 10^{6} \mathrm{~K}$ plasma component besides the $T \approx 1.5 \times 10^{6} \mathrm{~K}$ component known from previous PSPC observations. The existence of a high-temperature component, the observed limb-brightened X-ray surface brightness profile, and the observed level of $\mathrm{X}$-ray surface brightness cannot be satisfactorily explained by currently available models. Reducing heat conduction at the contact discontinuity may raise the central temperature and produce a limb-brightening; however, the expected X-ray surface brightness is still considerably higher than the observed surface brightness.
\end{abstract}

\section{Introduction}

The fast stellar wind of an early-type star sweeps up the surrounding medium into a dense shell, of which the central cavity is filled with a hot, tenuous, $\mathrm{X}$-ray emitting plasma. This structure is called a wind-blown bubble. Of all known bubbles, NGC 6888 is the most well-studied. The physical conditions of the hot interior of NGC 6888 have been analyzed by Wrigge at al. (1994) using ROSAT PSPC observations; a plasma temperature of $1.5 \times 10^{6} \mathrm{~K}$ is derived. This analysis might have missed a very hot plasma component because of the ROSAT PSPC's soft energy response and low spectral resolution. Therefore, we have obtained ASCA SIS observations of NGC 6888.

\section{Results and Discussion}

The ASCA-SIS and ROSAT-PSPC count rate spectra of the southern region of NGC 6888 are shown in Fig. 1a and a smoothed ASCA-SIS image of NGC 6888 is shown in Fig. 1b. We have fitted Raymond \& Smith's (1977; hereafter RS) optically-thin thermal-equilibrium plasma emission models to the combined SIS and PSPC spectra. We find that it is not possible to fit the combined spectra with a single-temperature plasma component. An additional, high-temperature component contributing fluxes to the higher energy 

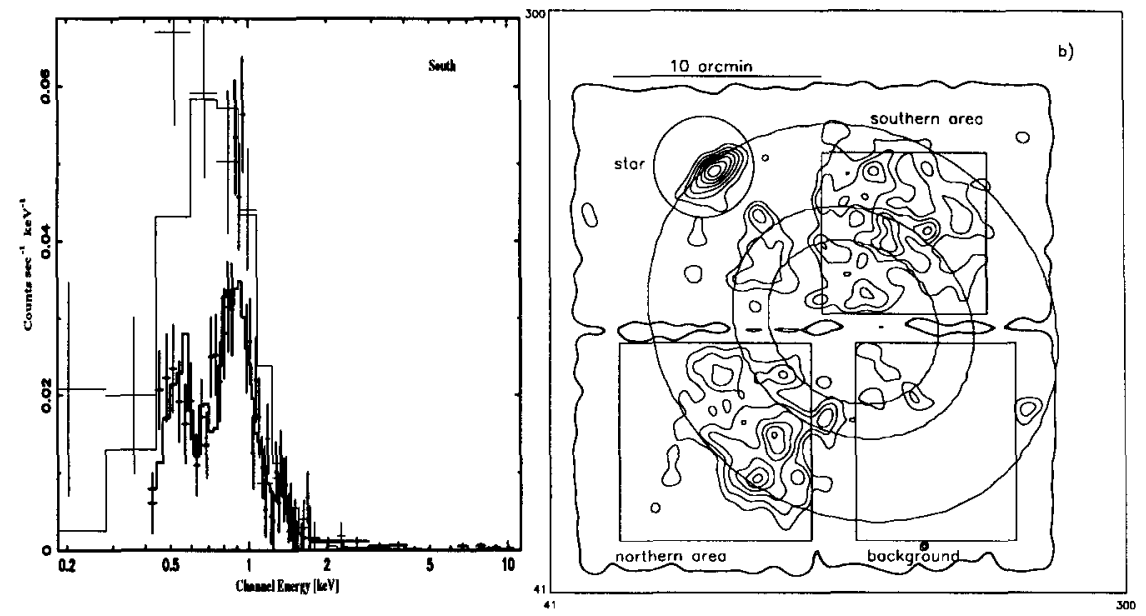

Fig. 1. (a) Count rate spectrum of the southern lobe of NGC 6888. The crosses are observation, and the fitted spectra are in lines. The ASCA-SIS spectrum is plotted in thick lines, and the ROSAT-PSPC spectrum in thin lines. (b) An ASCA-SIS image of NGC 6888 smoothed to $80^{\prime \prime}$ resolution. North is at $103^{\circ}$ counterclockwise from the top. The first contour level corresponds to $6.8 \times 10^{-4}$ counts s$^{-1} \operatorname{arcmin}^{-2}, 1.5 \sigma$ above the background of $\approx 4.8 \times 10^{-4}$ counts $\mathrm{s}^{-1} \operatorname{arcmin}^{-2}$. The step size between adjacent contours is $1 \sigma$. The contours running horizontally near the center of the image mark the gap between the SIS 0 and SIS 1 detectors. The bright point source to the west of NGC 6888 (at the upper left corner of the graph) is the nearby G8V star HD192020 unrelated to the nebula.

channels is needed to yield acceptable $\chi^{2}$ values. The two temperature components are $T \approx 1.5 \times 10^{6} \mathrm{~K}$ and $\approx 8 \times 10^{6} \mathrm{~K}$. The bubble structure of NCG 6888 has been analytically and numerically modeled by García-Segura \& Mac Low (1995a, 1995b; hereafter GM1 and GM2). Adopting the density and temperature structures provided by GM1 and adopting the plasma emission given by RS, we have modeled the X-ray spectra of NGC 6888 using the empirically determined physical parameters of the stellar wind and the expanding shell of NGC 6888 (see Wrigge et al. 1994). The modeled X-ray spectrum is then convolved with the instrumental response of ASCA-SIS for a direct comparison with the observed count rate spectrum. The modeled ASCA-SIS spectrum exhibits one peak, which can be reasonably well fitted by $\mathrm{a} \approx 1.5 \times 10^{6} \mathrm{~K}$ plasma emission component. This is clearly uncompatible with the observed spectrum, which requires an additional high-temperature component. We have also modeled the X-ray surface brightness profile of NGC 6888, using the above method. Despite the stronger emission expected in the dense outer region, the derived X-ray surface brightness profile shows a "centrally filled" appearance. The reason for the "centrally filled" appearance is that the softer X-ray emission from the cooler outer parts of the bubble is more heavily absorbed, as the absorption cross-section increases rapidly with 



Fig. 2. Expected surface brightness for models with decreased thermal conduction efficiency, i.e. increased temperature scale. The temperature inside the bubble is increased by a factor of 2 (upper left), 5 (upper right), 10 (lower left), and 20 (lower right) from the initial model.

decreasing plasma temperature, especially for temperatures below $1.5 \times 10^{6} \mathrm{~K}$. The "centrally filled" profile contrasts sharply the observed limb-brightened X-ray surface brightness profile of NGC 6888, shown in Fig. 1b. Lowering the foreground absorption column density $N_{\mathrm{H}}$ might help reconcile the difference between the expected and observed X-ray surface brightness profile. The Xray spectral fits give an $N_{\mathrm{H}}$ in the range of $(3 \ldots 3.8) \times 10^{21} \mathrm{~cm}^{-2}$, which is consistent with that derived from the optical extinction of NGC 6888 and a typical Galactic gas-to-dust ratio. Lowering this absorption column density by a factor of three increases the expected count rates by roughly a factor of three; however, the expected surface brightness profile still does not show significant limb-brightening as in the observed surface brightness profile. To produce an X-ray surface brightness profile that matches the observed profile, we have used the analytical model of GM1 with a range of shell mass, $M_{\text {shell }}$ $=4$ and $40 \mathrm{M}_{\odot}$, and shell expansion velocity, $v_{\exp }=75,100$, and $200 \mathrm{~km} \mathrm{~s}^{-1}$. However, none of these models can produce the observed limb-brightening. As the numerical calculations of GM2 reproduce the bubble dynamics of NGC 6888 remarkably well, we will consider GM2 models and assume that the bubble of NGC 6888 is pressure driven as in the "energy conserving" phase of expansion. We further assume that the temperature structure of the 
bubble interior is dominated by heat conduction across the contact discontinuity at the inner wall of the swept-up dense shell. If the efficiency of heat conduction at the interface is reduced, owing to a possible saturation of heat conduction (Dalton \& Balbus 1993) or the presence of magnetic fields, the temperature of the bubble interior will be higher. Thus, we consider changing the temperature structure of the bubble interior and examine its effect on the $\mathrm{X}$-ray surface brightness profile. We increase the central temperature by factors of $2,5,10$, and 20 from that of the initial model, which is described in the beginning of this section. Fig. (2a-d) show the X-ray surface brightness profiles for these four cases, respectively. The surface brightness profile changes to a limb brightened profile in Fig. $2 \mathrm{~b}$ with a central temperature of $4 \times 10^{7} \mathrm{~K}$. However, the overall surface brightness is considerably higher than that of the brightest peaks observed in NGC 6888. It thus seems that a simple increase of the temperature scale, as a result of lower efficiency of heat conduction, may be heading in the right direction for producing limb-brightening, but cannot reproduce the X-ray surface brightness profiles quantitatively.

\section{Conclusion}

Using ASCA-SIS observations, we have detected a high-temperature component of $T \approx 8 \times 10^{6} \mathrm{~K}$ besides the $T \approx 1.5 \times 10^{6} \mathrm{~K}$ plasma in NGC 6888 . In contrast to what is expected, the high temperature gas resides in the periphery of the nebula; no significant emission is detected from the central regions of the bubble. Both the northern part and the southern part show similar properties. Current models (GM1 and GM2) cannot reproduce the limb brightened appearance of NGC 6888. Varying input parameters within plausible ranges does not alleviate the problem. Ad hoc variations of the central plasma temperature of the bubble and a corresponding decrease in density may produce limb-brightened profiles; however, quantitative agreement is still not achieved. Only two Galactic bubbles have been detected in $\mathrm{X}$-rays, NGC 6888 and S308. As we discussed above, the X-ray spectra and morphology of NGC 6888 cannot be satisfactorily modeled, while S308 is too faint for detailed analysis of its spectral and morphological properties. Future deep X-ray observations of more wind-blown bubbles are needed to study the physical conditions of their hot interior.

\section{References}

Dalton W. W., Balbus S. A., 1993, ApJ 404, 625

García-Segura G., Mac Low M. - M., 1995a, ApJ 455, 145 (GM1)

García - Segura G., Mac Low M. - M., 1995b, ApJ 455, 160 (GM2)

Raymond J.C., Smith B.W., 1977, ApJS 35, 419 (RS)

Wrigge M., Wendker H.J., Wisotzki L., 1994, A\&A 286, 219 